\title{
A retrospective study on the use of post-operative colonoscopy following potentially curative surgery for colorectal cancer in a Canadian province Robert J Hilsden*1,2, Heather E Bryant ${ }^{2,3}$, Lloyd R Sutherland ${ }^{1,2}$, Penny MA Brasher ${ }^{2,3}$ and Anthony LA Fields 4
} \author{
${ }^{4}$ Department of Medicine University of Alberta, Edmonton, Alberta, Canada \\ Email: Robert J Hilsden* - rhilsden@ucalgary.ca; Heather E Bryant - heatherb@cancerboard.ab.ca; \\ Lloyd R Sutherland - lloyd@Iloydsutherlandgroup.com; Penny MA Brasher - littlep@cancerboard.ab.ca; \\ Anthony LA Fields - alaf@cancerboard.ab.ca \\ * Corresponding author
}

Address: ${ }^{1}$ Department of Medicine University of Calgary, Calgary, Alberta, Canada, ${ }^{2}$ Department of Community Health Sciences University of Calgary, Calgary, Alberta, Canada, ${ }^{3}$ Division of Epidemiology, Prevention and Screening Alberta Cancer Board, Calgary, Alberta, Canada and

Published: 19 April 2004

BMC Cancer 2004, 4:14

This article is available from: http://www.biomedcentral.com/I47/-2407/4//4

(c) 2004 Hilsden et al; licensee BioMed Central Ltd. This is an Open Access article: verbatim copying and redistribution of this article are permitted in all media for any purpose, provided this notice is preserved along with the article's original URL.
Received: 13 February 2004

Accepted: 19 April 2004

\begin{abstract}
Background: Surveillance colonoscopy is commonly recommended following potentially curative surgery for colorectal cancer. We determined factors associated with patients undergoing a least one colonoscopy within five years of surgery.

Methods: In this historical cohort study, data on 3918 patients age 30 years or older residing in Alberta, Canada, who had undergone a potentially curative surgical resection for local or regional stage colorectal cancer between 1983 and 1995 were obtained from the provincial cancer registry, ministry of health and cancer clinic charts. Kaplan-Meier estimates of the probability of undergoing a post-operative colonoscopy were calculated for patient, tumor and treatment-related variables of interest.
\end{abstract}

Results: A colonoscopy was performed within five years of surgery in 1979 patients. The probability of undergoing a colonoscopy for those diagnosed in the 1990s was greater than for those diagnosed earlier $(0.65$ vs $0.55, \mathrm{P}<0.000 \mathrm{I})$. The majority of the difference was seen at oneyear following surgery, consistent with changes in surveillance practices. Those most likely to undergo a colonoscopy were those under age 70 ( 0.74 vs 0.50 for those age $70-79, P<0.000 \mathrm{I})$, who underwent a pre-operative colonoscopy $(0.69$ vs $0.54, \mathrm{P}<0.000 \mathrm{I})$, and who underwent a resection with reanastomosis $(0.62$ vs 0.47 for abdominoperineal resection, $P<0.000 \mathrm{I})$ by a surgeon who performs colonoscopies $(0.68$ vs $0.54, \mathrm{P}<0.000 \mathrm{I})$.

Conclusions: The majority of patients undergo colonoscopy following colorectal cancer surgery. However, there are important variations in surveillance practices across different patient and treatment characteristics. 


\section{Background}

Colorectal cancer is a major cause of cancer-associated morbidity and mortality. Surgical resection is the treatment of choice for colorectal cancer and is often curative for early stage tumours. Patients with local lymph node metastases have a high rate of local and distant recurrence that can be reduced with adjuvant chemotherapy and/or radiotherapy. However, nearly $40-50 \%$ of patients who undergo curative intent surgery with or without adjuvant therapy will die in the following 5 years from local or distant recurrence[1]. In addition, patients who have had one colorectal cancer are also at increased risk of developing a second primary colorectal cancer[2,3].

Because of the risks of local or distant recurrence and new cancers, it is commonly recommended that patients undergo a variety of tests, including visualization of the remaining colon, over the months to years after their surgery to detect disease early, prior to the development of clinical symptoms, enhancing the success of effective, curative therapy [4-8]. With the development of fiber-optic colonoscopes in the 1970 's, most recommendations included colonoscopy as the primary method for examining the residual colon to detect intraluminal recurrence or new cancers.

Although controversy remains over how effective surveillance strategies are in reducing colorectal cancer mortality, some form of surveillance is almost uniformly recommended by experts, specialist organizations and cancer societies. However, there has been growing recognition that very intensive follow-up has not provided as substantial a survival benefit as initially hoped [9-11]. Yearly colonoscopy was commonly recommended in the $1980 s[8,12]$, but less frequent testing intervals were more commonly recommended in the early 1990s[7]. The combination of air-contrast barium enema and flexible sigmoidoscopy is often recommended as a reasonable alternative to colonoscopy. Guidelines differentiate between patients who undergo a pre-operative colonoscopy from those who do not: those who do not are recommended to undergo a colonoscopy within a year of surgery.

Variations in the practice patterns in the use of post-operative colonoscopy in the United States has been well documented [13-19]. In these studies, the use of colonoscopy varied across age groups, socio-economic status, race, levels of comorbidities, tumor location, stage, and SEER region. There is limited direct data on the use of colonoscopy in patients under age 65 , who comprise a significant proportion of colorectal cancer patients and who have potentially the most to gain from early detection of a recurrence or new cancer.
Examining practice patterns under different health care systems can provide further insights into the cause of practice variations. Canada has a similar standard of medical care but a markedly different system for funding health care. In contrast to the United States, Canadians are covered by universal, public health care insurance and do not incur out of pocket expenses for medically necessary physician or hospital services. Therefore, access in Canada is not limited by ability to pay for health care, but could be limited by availability of local resources.

The purpose of this study was to examine the use of postoperative colonoscopy in patients who had undergone a potentially curative resection for colorectal cancer between 1983 and 1995. Specifically, we wished to determine the likelihood that a patient would undergo a postoperative colonoscopy and whether this was affected by patient, tumour, treatment or surgeon characteristics. Based on previous research, we hypothesized that patients who were younger, who had more advanced tumours (Stage C: regional lymph node metastases) and who resided in urban areas would be more likely to undergo at least one post-operative colonoscopy. We also wished to examine the impact of gender, tumour site, type of surgery, preoperative colonoscopy and whether or not the surgeon performed colonoscopy on the probability of undergoing a post-operative colonoscopy. We examined whether practice patterns differed between the two large metropolitan areas, Edmonton and Calgary, where the majority of specialized medical care in the province is provided and that would be expected to have similar practice patterns. We also determined whether those who did not undergo a colonoscopy underwent a flexible sigmoidoscopy. Because we could not determine whether patients underwent a barium enema, by measuring flexible sigmoidoscopy we could provide an estimate of the maximum number of people who underwent alternative complete colonic evaluation.

\section{Methods}

In this historical cohort study, we linked the records of Alberta residents who had undergone a potentially curative resection for a first primary colorectal cancer identified from the Alberta Cancer Registry to Alberta Health \& Wellness administrative databases to determine the utilization of post-operative colonoscopy. Additional patient, tumor and treatment data was obtained by primary chart review of provincial cancer clinic charts. This study was approved by the Conjoint Health Research Ethics Board of the University of Calgary and the Research Ethics Committee of the Alberta Cancer Board.

\section{Subjects}

We included Alberta residents aged at least 30 years diagnosed with a first adenocarcinoma of the colon or rectum 
(International Classification of Diseases in Oncology topography codes C18.0 - C18.9, C19.9 or C20.9) between January 1, 1983 and December 31, 1995 who underwent a potentially curative resection and survived at least 270 days after their surgery. We excluded those with distant metastases, those who had a prior diagnosis of a non-colorectal invasive neoplasm, and those who were not eligible for coverage by the Alberta Health Care Insurance Plan (AHCIP) at the time of their diagnosis. We also excluded those with known inflammatory bowel disease, familial adenomatous polyposis or known hereditary cancer syndrome. Finally we excluded those who underwent a total proctocolectomy as their primary surgery, those with insufficient clinical information available to determine eligibility criteria and those who could not be successfully linked to Alberta Health \& Wellness databases.

Subjects were initially selected from the Alberta Cancer Registry. Following exclusion of those who did not meet eligibility criteria based on information available in the Cancer Registry, a random sample of patients was selected for full chart review. We used a sampling strategy that would result in approximately 4000 eligible subjects, but weighted towards those diagnosed in the late 1980s and early 1990s, the time period during which surveillance guidelines were changing and also a group who we believed would have the most complete follow-up data available. Therefore, all patients diagnosed from January 1, 1988 to December 31, 1992 were included and a 40\% random sample stratified by year of diagnosis and place of residence was chosen from subjects diagnosed in the remaining years.

\section{Data Sources and Variables Alberta Cancer Registry}

Coverage of registration for most cancers is at or above 95\%[20]. The Registry is linked monthly to Alberta Vital Statistics to detect the date and cause of death of registrants. Key demographic, tumor and treatment data is recorded routinely at the time of registration. The distance a patient resided from the nearest colonoscopy site was determined using the latitude and longitude of the patient's postal code of residence and postal code of the colonoscopy site[21].

\section{AHCIP Physicians Payments' Database}

This database was the primary source of data on colonoscopies and flexible sigmoidoscopies performed on patients prior to and after resection of their tumor. This database includes all payments for insured health care services provided to AHCIP registrants by in-province physicians. In Alberta during the years included in this study, fee-for-service physicians provided all endoscopy services. The date that a patient was no longer eligible for coverage by AHCIP was obtained from the AHCIP Population Registry.

All records for flexible sigmoidoscopy (code 01.24B) and colonoscopy (01.22) performed on patients from one year prior to the date of the diagnosis to March 31, 2000 were obtained. A specific billing code for performing an endoscopy via a stoma does not exist. Endoscopists tend to use the flexible sigmoidoscopy or colonoscopy fee codes depending on the extent of the exam. Only colonoscopies occurring before or on the date of diagnosis of cancer recurrence or new cancer were included as postoperative colonoscopies. The indication for the procedure is not recorded.

\section{Alberta Cancer Clinics' Clinical Charts}

All clinic charts maintained on a subject at any of the six provincial cancer clinics were reviewed to determine tumor stage (which is not present in the Registry database), type and date of primary operation, surgeon and disease outcomes. Tumor stage was determined from the review of the operative and pathology reports from a patient's primary surgery along with any other available clinical data (consult notes, diagnostic imaging reports). A surgeon was classified as performing colonoscopy if any procedure report of a colonoscopy performed by that surgeon was found in any patients chart (not just the charts of patients the surgeon operated on). A patient was considered to have undergone adjuvant there if they underwent pre-operative or post-operative chemotherapy or radiotherapy.

\section{Analysis \\ Missing data}

Because of the multiple, often overlapping data sources, complete data were available for most of the variables of interest. However, information was missing on tumor stage for 53 patients and place of residence for 9 . Forty-six of the 53 patients with missing tumor stage were assigned the stage recorded in their chart by their primary surgeon or oncologist. The remaining missing values for stage and place of residence were imputed based on their distribution in the rest of the study sample

\section{Statistical Analysis}

A survival analysis approach was taken for examining the risk of patients undergoing a first post-operative colonoscopy and to examine factors influencing this risk. Patients were censored at the date of any of the following events: diagnosis of cancer recurrence, loss of AHCIP coverage, or death. Kaplan-Meier estimates of the survival function were created. Differences in the survival function between different groups were examined graphically with KaplanMeier curves and tested using the Wald test. Next, extended Cox regression models were fit to examine the 


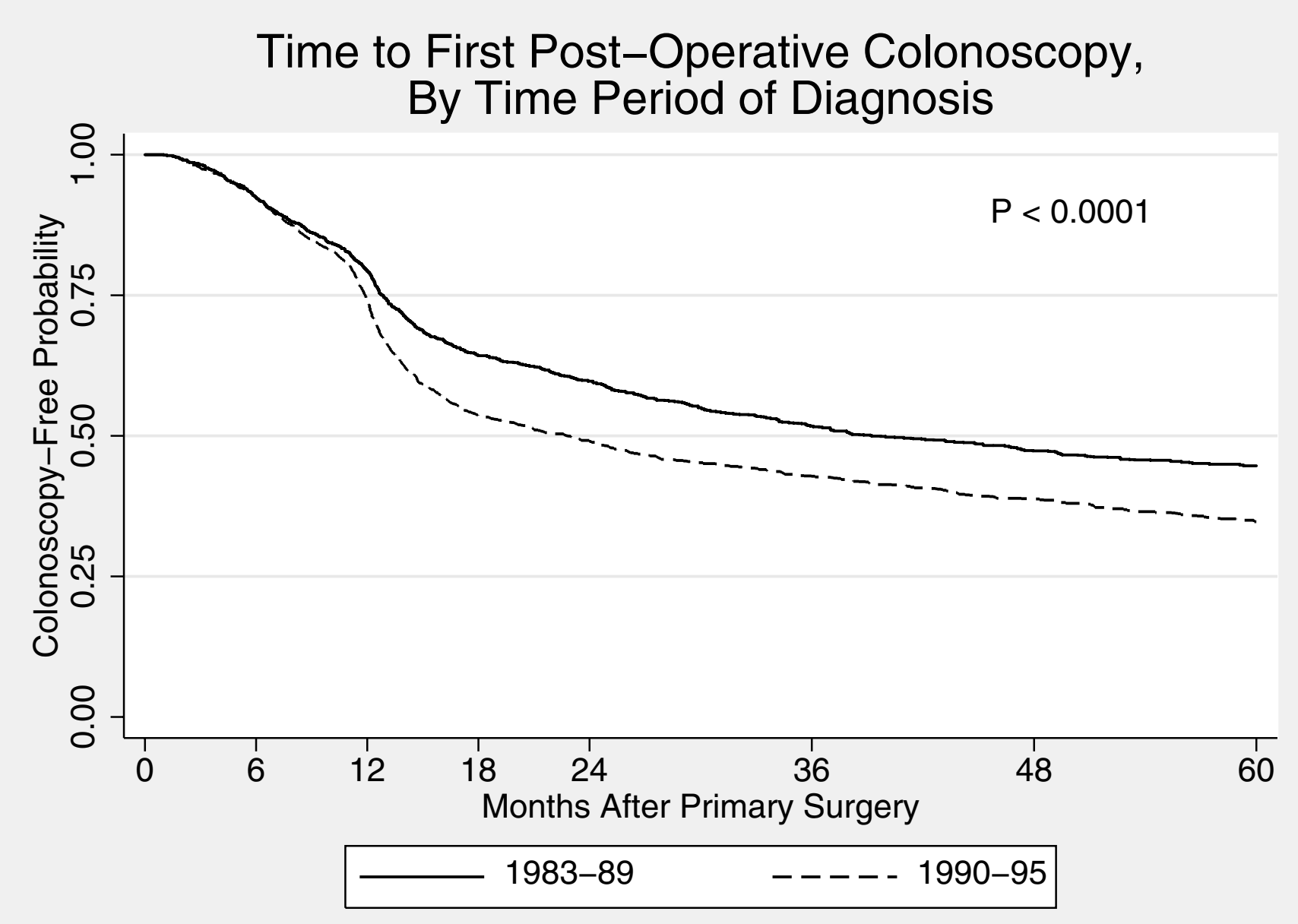

Figure I

Time to first post-operative colonoscopy stratified by time period of diagnosis. Kaplan-Meier survival curves of time to first post-operative colonoscopy for patients diagnosed from I983-1989 and 1990-1995. A divergence in the curves at I2 months is consistent with increased use of colonoscopy for post-operative surveillance.

independent effects of each predictor variable and to further examine confounding and interaction. Standard Cox models assume that the risk (hazard) of an event for one group is proportional to the risk of an event in the other group throughout the time period of interest. However, it was obvious from the examination of the Kaplan-Meier curves that the proportional hazards assumption was not met as often changes in the hazard of undergoing a postoperative colonoscopy only became apparent after the first 12 post-operative months (for example, see figure 1). We therefore used extended Cox regression methodology[22]. The survival history for each individual was split at the $12^{\text {th }}$ post-operative month. Models were then fit which generated two sets of coefficients for each variable included in the model: the first reflecting its effect over the first 12 months and the second its effect over the subse- quent 48 months. Variables were first examined in univariate and multivariate models of related variables (i.e. tumor characteristics). Variables significant at the 0.05 level were then included in multivariate models. The Akaike Information Criterion was used to assess the goodness of fit of models[23]. The statistical significance of parameters was tested using the Wald test. Robust estimates of the variance of each parameter were derived based on the method of Lin and Wei[24]. All analyses were performed using Stata version 7[25].

\section{Results}

From the Alberta Cancer Registry, 11494 patients with colorectal cancer were identified. Based on eligibility information available from the Registry, 3553 were excluded. Of the remaining 7941 potentially eligible 
Table I: Characteristics of patients included and excluded in full chart review

\begin{tabular}{lll}
\hline & Included & Excluded \\
\hline $\mathbf{n}(\%)$ & $1,924(40 \%)$ & $2,884(60 \%)$ \\
Sex: males & $1,066(55.4 \%)$ & $1,546(53.6 \%)$ \\
Age, years (mean) & 67.4 & 67.4 \\
Urban residence & $1,628(84.6 \%)$ & $2,431(84.3 \%)$ \\
Tumor site colon & $1,388(72.1)$ & $2,128(73.8 \%)$ \\
\hline
\end{tabular}

Table 2: Study sample characteristics and univariate associations with post-operative colonoscopy

\begin{tabular}{|c|c|c|c|}
\hline Variable & Sample distribution & Probability undergoing colonoscopy & $P$ value \\
\hline \multicolumn{4}{|l|}{ Sex } \\
\hline Male & $2188(56 \%)$ & 0.57 & \\
\hline Female & $1730(44 \%)$ & 0.63 & 0.021 \\
\hline \multicolumn{4}{|l|}{ Age group } \\
\hline $30-54$ & $559(14 \%)$ & 0.75 & \\
\hline $55-69$ & $1567(40 \%)$ & 0.74 & \\
\hline $70-79$ & 1161 (30\%) & 0.50 & \\
\hline$>80$ & $631(16 \%)$ & 0.28 & $<0.0001$ \\
\hline \multicolumn{4}{|l|}{ Place of residence } \\
\hline Urban & $582(15 \%)$ & 0.60 & \\
\hline Rural & $3336(85 \%)$ & 0.60 & 0.6 \\
\hline \multicolumn{4}{|l|}{ Tumor site } \\
\hline Colon & 2891 (74\%) & 0.54 & \\
\hline Rectum & $1027(26 \%)$ & 0.72 & $<0.0001$ \\
\hline \multicolumn{4}{|l|}{ Stage } \\
\hline 1 & $876(22 \%)$ & 0.65 & \\
\hline 2 & $1880(48 \%)$ & 0.57 & \\
\hline 3 & $1162(30 \%)$ & 0.63 & 0.0001 \\
\hline \multicolumn{4}{|l|}{ Pre-operative colonoscopy } \\
\hline Yes & $1646(42 \%)$ & 0.69 & \\
\hline No & $2272(58 \%)$ & 0.54 & $<0.0001$ \\
\hline \multicolumn{4}{|l|}{ Procedure type } \\
\hline Resection & $3363(86 \%)$ & 0.62 & \\
\hline Abdominoperineal resection & $555(14 \%)$ & 0.47 & $<0.0001$ \\
\hline \multicolumn{4}{|l|}{ Site of surgery } \\
\hline Calgary & $1270(32 \%)$ & 0.58 & \\
\hline Edmonton & $1733(44 \%)$ & 0.63 & \\
\hline Other Major City & $607(15 \%)$ & 0.60 & \\
\hline Other & $308(8 \%)$ & 0.55 & $<0.0001$ \\
\hline \multicolumn{4}{|l|}{ Adjuvant therapy } \\
\hline Yes & $1049(27 \%)$ & 0.72 & \\
\hline No & 2869 (93\%) & 0.57 & $<0.0001$ \\
\hline \multicolumn{4}{|l|}{ Cancer clinic visits } \\
\hline None & 1691 (43\%) & 0.52 & \\
\hline $\mathrm{I}-4$ & $1434(37 \%)$ & 0.63 & \\
\hline$\geq 5$ & $793(20 \%)$ & 0.74 & $<0.0001$ \\
\hline \multicolumn{4}{|l|}{$\begin{array}{l}\text { Surgeon performs } \\
\text { colonoscopies }\end{array}$} \\
\hline Yes & I $838(47 \%)$ & 0.68 & \\
\hline No & $2080(53 \%)$ & 0.54 & $<0.0001$ \\
\hline
\end{tabular}


patients, 5057 were selected for chart review. Table 1 compares basic demographic and tumor characteristics of those who were and were not selected for chart review. Of those who underwent chart review, 3918 patients met all inclusion criteria and were included in the final sample, 1826 diagnosed from 1983 - 1989 and 2092 diagnosed from 1990 - 1995. The main reasons for exclusion based on Cancer Registry information were previous or synchronous non-colorectal cancer $(\mathrm{n}=1147)$, not eligible for Alberta Health Care coverage $(n=215)$ and ineligible tumor morphology $(n=162)$. The main reasons for exclusion based on information obtained at chart review were distant metastases $(n=620)$, endoscopic polypectomy only treatment $(\mathrm{n}=140)$, and tumor not resected $(\mathrm{n}=$ 133).

The demographic, tumor and treatment characteristics of the sample are shown in Table 2. The proportions of patients residing within 10 kilometers, 10 - 99 kilometers and 100 kilometers or more from the nearest colonoscopy site were $68 \%, 18 \%$ and $14 \%$, respectively. The five-year survival for the entire sample was $66 \%$ and was $83 \%, 68 \%$ and $48 \%$ for those with tumor stage 1, 2 and 3, respectively. Of the 1829 deaths, 1105 (60\%) were due to colorectal cancer, $116(6 \%)$ due to other new non-colorectal cancers and 608 (33\%) due to non-cancer causes.

\section{First Post-Operative Colonoscopy}

At least one colonoscopy was performed within five years of the primary surgery in 1979 (51\%) patients. Patients who were diagnosed from 1990 - 1995 were more likely to undergo a colonoscopy than those diagnosed from 1983 - 1989 (Figure 1: 5-year cumulative probability 0.65 vs. $0.55, P<0.0001)$. The difference in colonoscopy rates between the two groups is primarily due to an increased likelihood of those diagnosed from 1990 - 1995 undergoing a colonoscopy at around the 12-month mark.

Table 2 also shows the five-year probability of undergoing a colonoscopy for different patient, tumor, treatment and surgeon characteristics. Tumor site (rectal versus colonic) was not associated with the probability of undergoing a post-operative colonoscopy after adjusting for the effect of type of surgical procedure (resection with reanastomosis versus abdominoperineal resection).

The influence of some of these factors on the probability of a patient undergoing a post-operative colonoscopy depended on the time period that the person was diagnosed. For those diagnosed from 1983 - 1989, women were less likely than men to undergo a post-operative colonoscopy (5-year cumulative probability 0.49 versus 0.61). However, for those diagnosed in the later time period, no difference was apparent (0.66 versus 0.65 ). Place of residence was a significant predictor of the prob- ability of undergoing a colonoscopy for those diagnosed in the early time period but not in the later. Pre-operative colonoscopy and location (city) of surgery were significant predictors in both time periods. Figures 2 \&3 show Kaplan-Meier curves of time to first post-operative colonoscopy for patients who underwent surgery in Calgary and Edmonton stratified by time period of diagnosis. In the early time period, patients in Calgary were much less likely to undergo a colonoscopy than patients in Edmonton ( 0.49 vs. 0.57$)$, but this was no longer the case in the later time period ( 0.66 vs. 0.63$)$. This change is due to the marked increase use of colonoscopy at the 12-month post-surgery mark in those patients who underwent surgery in Calgary (Figure 2). In contrast, there was little change in the use of colonoscopy in Edmonton over the time period of this study (Figure 3 ).

To further explore potential confounding and interaction among the variables, extended Cox proportional hazard models were fit. In these models the data was split into two time periods: post-operative months $1-12$ and $13-$ 60 . The final model produces one set of hazard ratios reflecting the association between the variable and the probability of undergoing a colonoscopy for the first 12 months following surgery and a second set for the next 48 months. The results of the model are shown in Tables 3 and 4 . Table 3 shows the results for variables that are not associated with a time period of diagnosis interaction and Table 4 for those that are. The majority of the hazard ratios shown in Tables 3 and 4 do not substantially differ from those obtained from univariate models (data not shown). However, the hazard ratios associated with site of surgery, specifically those associated with the comparison of Calgary to Edmonton did change. When site of surgery was examined alone, patients in Calgary diagnosed from 1990 - 1995 were shown to have a hazard ratio of 1.31 compared with Edmonton during the follow-up months 13 60 . However, in the multivariable model this hazard ratio is now 1.01. This change occurs only when the cancer clinic visits variable is added to the model. Patients who underwent surgery in Calgary on average had more cancer clinic visits than patients who underwent surgery in Edmonton (mean 3.7 vs. 1.9).

\section{Post-Operative Flexible Sigmoidoscopy}

Only 224 (11.6\%) patients who did not undergo a postoperative colonoscopy underwent a post-operative sigmoidoscopy. A similar proportion (10.6\%) of those who did undergo a post-operative colonoscopy also underwent a flexible sigmoidoscopy prior to their colonoscopy. For those who did not undergo a post-operative colonoscopy, undergoing a post-operative flexible sigmoidoscopy was not associated with their age or sex, tumor stage, number of clinic visits, whether they underwent a preoperative colonoscopy or whether their surgeon per- 


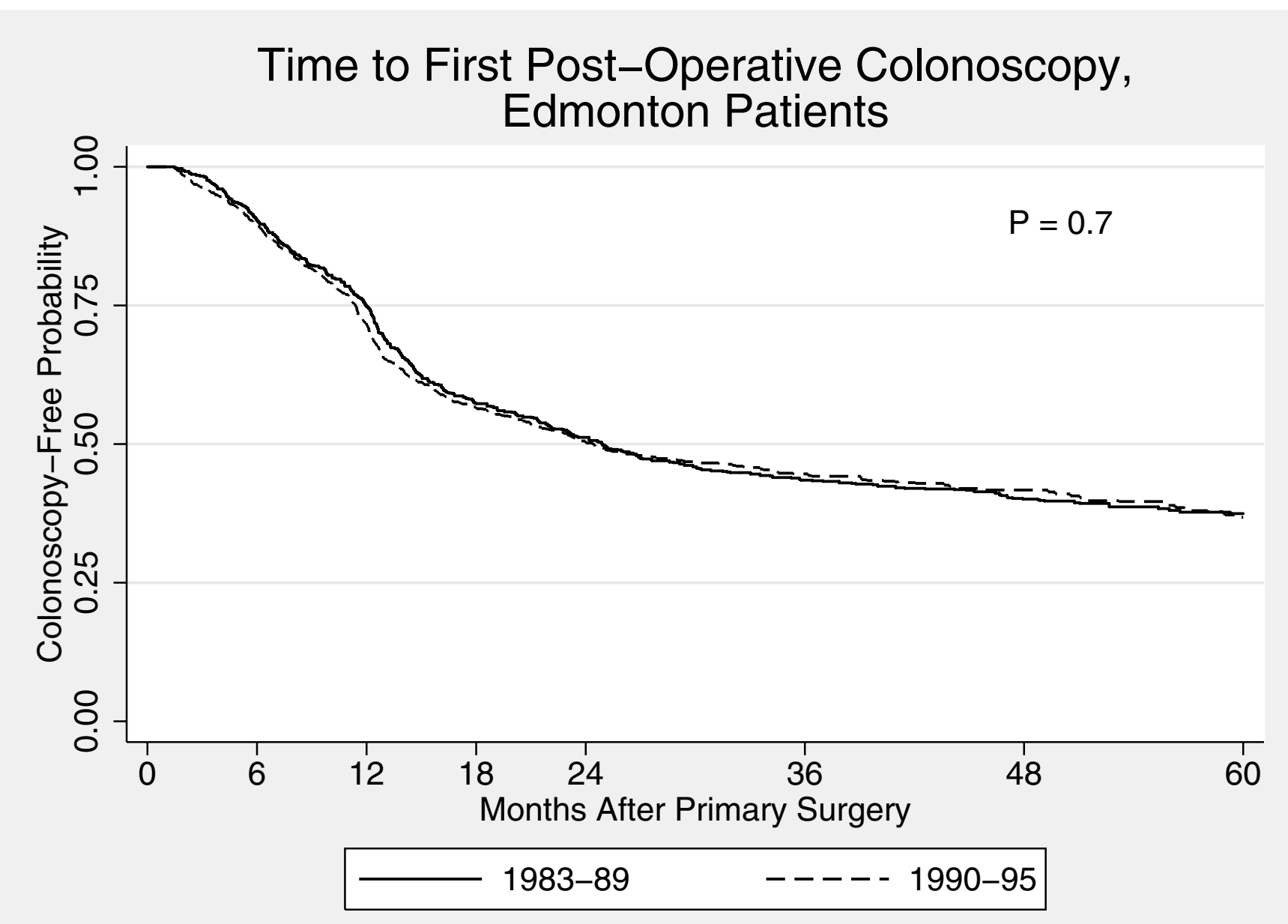

Figure 2

Time to first post-operative colonoscopy for Edmonton patients Kaplan Meier curve of time to first post-operative colonoscopy for patients who underwent primary cancer surgery in Edmonton, stratified by time period of diagnosis. No change in practice patterns are evident from the curves for the two time periods.

formed colonoscopies. There was, however, a strong association with procedure type and where they underwent surgery. Only $3(0.9 \%)$ who underwent an abdominoperineal resection underwent a flexible sigmoidoscopy compared with $2221(13.8 \%)$ of those who underwent a resection. Those who underwent their surgery in smaller cities (Other city) were twice as likely to undergo a flexible sigmoidoscopy $(22.7 \%)$ than those who underwent their surgery in either of the two large cities or elsewhere $(\mathrm{p}<$ 0.0005).

\section{Discussion}

In this study, we showed that there have been important variations in the use of colonoscopy following potentially curative surgery in Alberta. Furthermore, there have been significant changes in the use of the test over the past two decades. Based on the existing literature, we had hypothesized that younger patients, those with more advanced tumors and those residing in urban areas would be more likely to undergo at least one post-operative colonoscopy.

When examining the Kaplan-Meier curves for time to first post-operative colonoscopy, a clear change in the hazard of undergoing a colonoscopy is seen at around the oneyear mark following surgery. This is most consistent with increased use for surveillance purposes, as one would not expect such a dramatic increase use in colonoscopy at one year following the date of surgery just for the investigation of symptoms. Furthermore, when examining the effect of different variables on the probability of undergoing a 


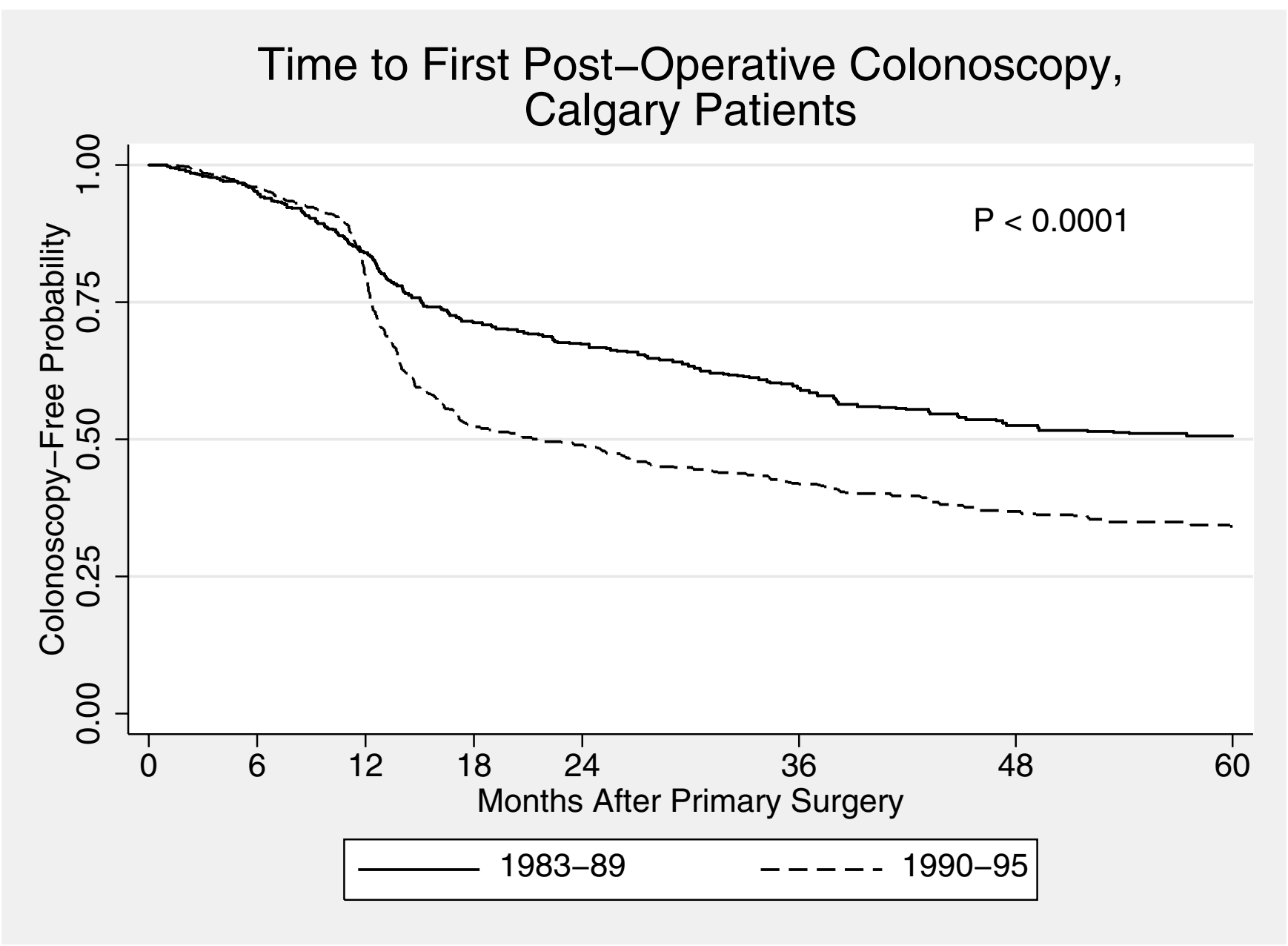

Figure 3

Time to first post-operative colonoscopy for Calgary patients Kaplan Meier curve of time to first post-operative colonoscopy for patients who underwent primary cancer surgery in Calgary, stratified by time period of diagnosis. A major change in practice patterns is evident as patients diagnosed from 1990 - 1995 are much more likely to undergo a post-operative colonoscopy, primarily due to increased use of colonoscopy at one year after surgery.

post-operative colonoscopy, many of the differences are only seen or become most pronounced at around the oneyear mark. This suggests that differences between groups are primarily due to differences in surveillance practices.

Just over $50 \%$ of all patients underwent at least one postoperative colonoscopy, which is in keeping with testing rates reported in Medicare patients by Cooper[26]. However, testing did increase in the 1990s, as the probability of a patient diagnosed in the 1990s undergoing at least one post-operative colonoscopy was 0.65 compared with 0.55 for a patient diagnosed in the 1980s. This difference becomes apparent at the one-year post-operative mark, thus indicating that differences in use between the two time periods is due to changes in surveillance practices.
Several other variables were shown to be associated with the probability of undergoing a first post-operative colonoscopy, including age, undergoing a preoperative colonoscopy, type of surgery, number of cancer clinic visits, whether the surgeon performs colonoscopies and where the patient underwent their cancer surgery. Surveillance guidelines do not base recommendations for screening on the majority of the variables that were found to be associated with post-operative colonoscopy in this study. Certainly some of these variables would be expected to be associated with the likelihood of undergoing a post-operative colonoscopy, even if they were not explicitly stated in surveillance recommendations. Older patients, especially those with significant comorbidities, would have less to gain from surveillance as their survival may be 
Table 3: Extended Cox Regression Model: First post-operative colonoscopy variables not associated with time period of diagnosis

\begin{tabular}{|c|c|c|}
\hline & Month I - I 2 & Month $13-60$ \\
\hline & HR (95\% Cl) & HR (95\% Cl) \\
\hline \multicolumn{3}{|c|}{ Age Group (vs $30-54)$} \\
\hline $55-69$ & $0.77(0.63-0.93)$ & $0.98(0.81-1.19)$ \\
\hline $70-69$ & $0.48(0.38-0.60)$ & $0.53(0.43-0.66)$ \\
\hline $80+$ & $0.14(0.10-0.21)$ & $0.29(0.21-0.38)$ \\
\hline \multicolumn{3}{|l|}{ Procedure type } \\
\hline APR vs Resection & $0.29(0.22-0.40)$ & $0.71(0.59-0.86)$ \\
\hline \multicolumn{3}{|c|}{ Cancer clinic visits (vs 0 ) } \\
\hline $1-4$ & $1.09(0.92-1.30)$ & $1.39(1.19-1.63)$ \\
\hline$\geq 5$ & $1.13(0.92-1.39)$ & $1.69(1.39-2.06)$ \\
\hline \multicolumn{3}{|c|}{ Surgeon performs colonoscopy } \\
\hline Yes vs No & $1.85(1.59-2.15)$ & $1.38(1.21-1.59)$ \\
\hline
\end{tabular}

Table 4: Extended Cox Regression Model: first post-operative colonoscopy variables associated with time period of diagnosis

\begin{tabular}{|c|c|c|}
\hline & Month I - I 2 & Month I3-60 \\
\hline & HR (95\% Cl) & HR (95\% Cl) \\
\hline \multicolumn{3}{|l|}{ Sex (M vs F) } \\
\hline $1983-1989$ & $0.88(0.69-1.11)$ & $1.24(1.01-1.53)$ \\
\hline $1990-1995$ & $0.92(0.76-1.11)$ & $0.87(0.73-1.05)$ \\
\hline \multicolumn{3}{|c|}{ Pre-operative colonoscopy (yes vs no) } \\
\hline $1983-1989$ & $1.52(1.20-1.91)$ & $1.57(1.29-1.93)$ \\
\hline $1990-1995$ & $0.88(0.73-1.06)$ & $1.46(1.22-1.75)$ \\
\hline \multicolumn{3}{|c|}{ Site of surgery (vs. edmonton) } \\
\hline \multicolumn{3}{|c|}{$1983-1989$} \\
\hline Calgary & $0.56(0.43-0.74)$ & $0.61(0.48-0.78)$ \\
\hline Other major city & $0.71(0.50-1.02)$ & $0.69(0.50-0.95)$ \\
\hline Other & $0.74(0.46-1.20)$ & $0.66(0.46-0.96)$ \\
\hline \multicolumn{3}{|l|}{$1990-1995$} \\
\hline Calgary & $0.54(0.43-0.68)$ & $1.08(0.87-1.33)$ \\
\hline Other major city & $1.06(0.82-1.37)$ & $1.34(1.04-1.73)$ \\
\hline Other & $0.78(0.53-1.15)$ & $0.97(0.66-1.42)$ \\
\hline
\end{tabular}

more limited by their other medical conditions. Unfortunately, we could not accurately measure comorbidities, especially in a longitudinal fashion over the course of the patients follow-up, to determine what impact concomitant medical problems had on the likelihood of undergoing surveillance in more elderly patients.

Our data did not support our second hypothesis that those with more advanced tumors would be more likely to undergo a colonoscopy. Although we saw some differences between those with different stage tumors in the probability of undergoing a colonoscopy this was not a linear trend. Those with Stage A and C tumors were more likely to undergo a colonoscopy than those with Stage B. This association was not seen in the multivariate analysis.

A pre-operative colonoscopy would not be expected to affect the likelihood of undergoing a post-operative one, although it would be expected to affect the timing. In general, guidelines have recommended a colonoscopy within one year for those patients who did not undergo a preoperative colonoscopy. However, patients who did not undergo a pre-operative colonoscopy were less likely to undergo a post-operative colonoscopy within the first five post-operative years and the expected pattern of undergoing an early post-operative colonoscopy was not seen. In fact, in those who did not undergo a pre-operative colon- 
oscopy there was little change in the Kaplan-Meier curve at the one-year mark suggesting that there was little use of surveillance colonoscopy in this group.

Patients may not undergo a pre-operative colonoscopy if they presented with a complicated tumor, either perforated or obstructed. Alternatively they may not undergo a colonoscopy if the tumor is visualized by barium enema or is located in the rectum and visualized by sigmoidoscopy or proctoscopy. However, these patients, potentially, have the most to gain from a post-operative colonoscopy to detect metachronous polyps or cancers. Preferential use of barium enema could be the result of physician preferences or lack of local colonoscopy resources.

One of the most interesting differences in this study is that seen between patients who were treated in Edmonton and Calgary. These two cities have the two largest cancer clinics and the largest number of surgical and cancer specialists. Therefore, one might anticipate quite similar practice patterns. However, there were marked differences in the use and timing of post-operative colonoscopy. Patients diagnosed in the 1980s who underwent their cancer surgery in Edmonton were much more likely to undergo a post-operative colonoscopy than those patients who underwent their surgery in Calgary. A possible reason for the higher frequency of post-operative colonoscopy in Edmonton was the establishment of a postoperative surveillance colonoscopy service at the Cross Cancer Institute circa 1980. A similar program did not exist in Calgary. However, in the 1990s, this difference was no longer apparent. There had been a marked change in the use of colonoscopy in Calgary, essentially all due to increased use at the one-year post-operative mark. In contrast, no change was seen in the use of colonoscopy in Edmonton over the 13 years of this study.

Differences between these two cities could also be due to differences in colonoscopy capacity, differences in physician practice patterns or a combination of the two. In general, the endoscopy resources, in terms of endoscopists and endoscopy sites, would have been similar int the two cities. However, recent work has shown that in the early 1990 's, only one-half the number of colonoscopies were performed in Calgary as were performed in Edmonton despite a similar population[27]. This difference had markedly narrowed by 2000 . Therefore, if this trend was also present in the 1980's, it is reasonable to assume that colon cancer patients in Calgary had less access to colonoscopy, presumably due to different physician practice patterns.

Our third hypothesis was that those residing in rural areas would be less likely to undergo colonoscopy. However, we could not find evidence supporting this. This may be due to marked increase in colonoscopy resources outside of the two major urban areas[27]. The majority of patients resided within $10 \mathrm{~km}$ of the nearest colonoscopy site, and only $14 \%$ lived more than $100 \mathrm{~km}$ away.

We could not collect data on other post-operative testing, such as CEA, barium enemas or CT scans. The existing administrative databases, either do not or incompletely include records on these tests. Therefore, we cannot determine whether patients underwent alternative tests, such as double-contrast barium enema, instead of colonoscopy or if not undergoing a colonoscopy was associated with not undergoing other post-operative surveillance testing, such as CEA. An alternative to colonoscopy for complete colonic examination is the combination of flexible sigmoidoscopy and air-contrast barium enema. We did measure flexible sigmoidoscopy use and found that only $11.6 \%$ of patients who did not undergo a post-operative colonoscopy underwent a flexible sigmoidoscopy. Therefore, flexible sigmoidoscopy alone or combined with a barium enema was not commonly used as an alternative to colonoscopy.

The value of post-operative surveillance, including colonoscopy continues to be debated. No randomized controlled trial has been conducted specifically addressing the issue of colonoscopic surveillance. However, three published randomized studies of intensive follow-up found no benefit from a follow-up program that included regular colonoscopic examination $[10,11,28]$. Kjeldsen et al[10] in a Danish trial found no benefit to an intensive follow-up regimen that included colonoscopy every six months. However, all patients underwent a post-operative colonoscopy prior to entry into the study. In a Finnish trial, Makela et al[28] randomized patients to intensive versus conventional follow-up and could not demonstrate a survival benefit or increased resectability despite recurrences being identified earlier in the intensive surveillance group. Intensive follow-up included yearly colonoscopy. However, regular colonic testing also occurred in the conventional follow-up, including annual air-contrast barium enemas and fecal occult blood testing. Finally, Schoemaker et al[11] included fecal occult blood testing every three months for two years and then every six months for three years in their conventional follow-up group. The intensive follow-up group underwent yearly colonoscopy. Seventy-two colonoscopies were performed on the 158 patients in the conventional treatment group compared with 577 in the 167 patients in the intensive treatment group. The authors noted that most metachronous and locally recurrent carcinomas in each group were detected by means other than colonoscopy. A statistically significant survival advantage could not be demonstrated for the intensive follow-up group. 


\section{Conclusions}

In conclusion we found important variations in the use of colonoscopy following potentially curative colorectal cancer surgery inpatients diagnosed in the province of Alberta from 1983 to 1997 . The majority of factors that were associated with these variations would not have been predicted based on prevailing surveillance guidelines. Overall, there was evidence of increased use of at least one post-operative colonoscopy over the time course of the study. Given that surveillance colonoscopy will remain a recommended surveillance strategy, even in the absence of RCT level evidence supporting its efficacy, it is imperative that those physicians managing patients with colorectal cancer endeavor to insure that all patients who may potentially benefit have access to the procedure. Improved communication between those who provide the majority of the post-operative care and those who provide endoscopic services may be required.

\section{Competing Interests}

None declared.

\section{Authors' Contributions}

The study was completed as part of the requirements for doctoral degree for Dr. Hilsden from the Department of Community Health Sciences, University of Calgary. Dr. Brant acted as Dr. Hilsden's supervisor and the other authors were members of the supervisory committee. Dr. Hilsden conceived of the project and with Dr. Bryant and the other authors developed the research protocol. Dr. Hilsden oversaw all aspects of the conduct of the study and wrote the first draft of the manuscript. All authors participated in the revision of the manuscript and approved the final version.

\section{Acknowledgements}

This project was supported by an operating grant from the Alberta Heritage Foundation for Medical Research. Dr. Hilsden was supported by the Medical Research Council of Canada and the Alberta Heritage Foundation for Medical Research.

\section{References}

I. Moertel CG, O'Fallon JR, Go VL, O'Connell MJ, Thynne GS: The preoperative carcinoembryonic antigen test in the diagnosis, staging, and prognosis of colorectal cancer. Cancer 1986, 58:603-610

2. Cali RL, Pitsch RM, Thorson AG: Cumulative incidence of metachronous colorectal cancer. Dis Colon Rectum 1993, 36:388-393.

3. Heald RJ: Synchronous and metachronous carcinoma of the colon and rectum. Ann R Coll Surg Engl 1990, 72:172-174.

4. Enker WE, Kramer RG: The follow-up of patients after definitive resections for large bowel cancer. World J Surg 1982, 6:578-584.

5. Langevin JM, Wong WD: What is the appropriate follow-up for the patient with colorectal cancer? Can J Surg 1985, 28:424-428.

6. Rocklin MS, Slomski CA, Watne AL: Postoperative surveillance of patients with carcinoma of the colon and rectum. American Surgeon 1990, 56:22-27.

7. Surgeons American Society of Colon and Rectal: Practice parameters for the detection of colorectal neoplasms. Dis Colon Rectum 1992, 35:389-390.
8. Devasa JM, Morales V, Enriques JM, Nuno J, Camunas J, Hernandez MJ, Avila C: Colorectal cancer. The bases for a comprehensive follow-up. Dis Colon Rectum 1987, 31:636-652.

9. Moertel CG, Fleming TR, Macdonald JS, Haller DG, Laurie JA, Tangen $C$ : An evaluation of the carcinoembryonic antigen (CEA) test for monitoring patients with resected colon cancer. JAMA 1993, 270:943-947.

10. Kjeldsen BJ, Kronborg O, Fenger C, Jorgensen OD: A prospective randomized study of follow-up after radical surgery for colorectal cancer. Br J Surg 1997, 84:666-669.

II. Schoemaker D, Black R, Giles L, Toouli J: Yearly colonoscopy, liver CT, and chest radiography do not influence 5-year survival of colorectal cancer patients. Gastroenterology 1998, II 4:7-I4.

12. Larson GM, Bond SJ, Shallcross C, Mullins R, Polk HC: Colonoscopy after curative resection of colorectal cancer. Arch Surg 1986, 1 21:535-540.

13. Johnson FE, Longo WE, Vernava AM, Wade TP, Coplin MA, Virgo KS: How tumor stage affects surgeons' surveillance strategies after colon cancer surgery. Cancer 1995, 76: I 325-1329.

14. Virgo KS, Wade TP, Longo WE, Coplin MA, Vernava AM, Johnson FE: Surveillance after curative colon cancer resection: practice patterns of surgical subspecialists. Ann Surg Oncol 1995, 2:472-482.

15. Cooper GS, Yuan Z, Chak A, Rimm AA: Geographic and patient variation among medicare beneficiaries in the use of followup testing after surgery for nonmetastatic colorectal carcinoma. Cancer 1999, 85:2124-2131.

16. Ellison GL, Warren JL, Knopf KB, Brown ML: Racial differences in the receipt of bowel surveillance following potentially curative colorectal cancer surgery. Health Services Research 2003, 38:t-903.

17. Elston Lafata J., Cole Johnson C., Ben Menachem T, Morlock RJ: Sociodemographic differences in the receipt of colorectal cancer surveillance care following treatment with curative intent. Medical Care 200I, 39:36I-372.

18. Rulyak SJ, Mandelson MT, Brentnall TA, Rutter CM, Wagner EH: Clinical and sociodemographic factors associated with colon surveillance among patients with a history of colorectal cancer. Gastrointestinal Endoscopy 59 (2):239 -47, 2004

19. Knopf KB, Warren JL, Feuer EJ, Brown ML: Bowel surveillance patterns after a diagnosis of colorectal cancer in Medicare beneficiaries. Gastrointestinal Endoscopy 200 I, 54:563-57I.

20. Brasher PMA: Alberta Cancer Registry. 1992 Annual Report. Alberta, Alberta Cancer Board; 1995.

21. Ng E, Wilkins R, Perras A: How far is it to the nearest hospital? Calculating distances using the Statistics Canada Postal Code Conversion File. Health Rep 1993, 5:179-188.

22. Collett D: Some additional topics. Modelling survival data in medical research London, Chapman \& Hall; 1993:267-273.

23. Collett $D$ : Modelling survival data. Modelling survival data in medical research London, Chapman \& Hall; 1993:267-273.

24. Lin DY, Wei LJ: The robust inference for the Cox proportional hazards model. Journal of the American Statistical Association 1989, 84:1074-1078.

25. StataCorp: Stata Statistical Software: Release 7.0. Edited by: Stata Corporation. College Station, Tx; 200I.

26. Mindell WR, Vayda E, Cardillo B: Ten-year trends in Canada for selected operations. Can Med Assoc ] 1982, I27:23-27.

27. Hilsden RJ: Patterns of use of flexible sigmoidoscopy, colonoscopy and gastroscopy: A population-based study in a Canadian province. Can / Gastroenterol 2004, I 8:213-219.

28. Makela JT, Laitinen SO, Kairaluoma MI: Five-year follow-up after radical surgery for colorectal cancer. Results of a prospective randomized trial. Arch Surg 1995, 130:1062-1067.

\section{Pre-publication history}

The pre-publication history for this paper can be accessed here:

http://www.biomedcentral.com/1471-2407/4/14/prepub 